\title{
Dynamic Evaluation of English Second Classroom Based on Schema Theory
}

\author{
Xijuan Chen \\ Nanchang Institute of Science and Technology \\ Jiangxi, China 330108
}

\begin{abstract}
Based on the cognitive context, this article researches on the second English class through schema theory. According to investigation and analysis, the evaluation method to university English second classroom can be improved. Based on the combination of formative evaluation and summative evaluation, the dynamic evaluation method can make up for the inadequacy of single summative evaluation.
\end{abstract}

Keywords-schema theory; second classroom; summative evaluation; formative evaluation

\section{INTRODUCTION}

The process of language learning is actually a process of cognition. In recent years, with the development of cognitive linguistics, schema theory has been attracted more and more attention to. Cook defined schema as a kind of "knowledge structure" existing in the brain as a whole and the specialized knowledge, which is composed of many similar events or information [1]. Once a schema is formed, communicative information will tend to be the schema. Schema theory shows that the human brain has all kinds of knowledge about the world which is stored in the form of schema. Language comprehension is a process of combining the known schema with the received information to establish a new schema, which is a process of activation, or to construct a proper schema and fill with new information, a process of interaction between background knowledge and new information. The input information must be matched with the schema, which can complete a series of information processing. The low-level schema gradually changes through assimilation, coordination and balance to the high-level. Foreign language learning is a new scheme formation process combined by the knowledge in the books and from the teachers and the existing old scheme [2]. From the cognitive perspective, this is actually the process of learners' recognition, processing and understanding of the learning materials.

\section{The SECOND ClassRoOM OF COLLEGE ENGLISH}

\section{A. The Necessity of the Second Classroom of College} English

The goal of College English teaching mainly focuses on the talent training, highlighting the practical ability of language application, emphasizing the language foundation and language application ability[3]. But, due to the limited time and the expanding college enrollment in recent years, students practice opportunity is extremely limited in the traditional class teaching mode, which can not meet the students' comprehensive language application. Therefore, teachers need to use the second classroom to carry out a great deal of language practice activities.

\section{B. The present situation of the second classroom of college English}

At present, there are different second classroom for English teaching in many colleges and universities. Such as morning reading in English, English salon, the weekly English corner, regular English broadcast to all students every day, and organized all kinds of English competitions, for example, English word contest, drama performance, English speech contest and so on. Students are likely to enjoy watching the film at the weekend, and they love Christmas, Valentine's Day, April fool's day, Thanksgiving Day. It shows that most of the students tend to participant in the second class in high enthusiasm.

Most students think that the selected theme in the second class can arouse their interest, and the theme can be fully connected with the learning in the first class. Some students choose the second class for the relatively strong language atmosphere. The most important reason for students who are not willing to participate in the second English class is because they can not understand, can not speak.

Students suggest that the teachers can guide the method of learning, stimulate their interest in learning English, and enhance their confidence in English learning. Classroom activities should be carried out step by step, and be connected with the teaching in the first class. To consolidate the learned knowledge, the activities can contact the reality. The selected content should conform to the actual level of the students. So they can have the opportunity to speak, understand, and cultivate a sense of the English language learning.

\section{THE ENLIGHTENMENT OF SCHEMA RELEVANCE THEORY TO THE SECOND ENGLISH CLASS}

\section{A. Constructing Cognitive Context, Searching for Optimal Relevance and Activating Schemata}

According to the cognitive theory, schema is the cognitive foundation. After obtaining the new information, the information will be to re-organized understood and formed in the brain [4]. After the input, absorption and 
understanding, and the brain will process the schema matching, and can play a role in complete acceptance, understanding, decoding and storage. Students in the second class show a negative state, because the relative cognitive context is not constructed for the students themselves, which can not activate the relevant schemata in their mind, so it can not stimulate their the interest in learning.

So how to construct the cognitive context and activate the relevant schemata as far as possible has become the core task of the second class. In the construction of cognitive context, the input object, image, character, voice and other physical environment can maximize students' cognitive schemata in the brain. So, the activation of irrelevant schemata is unable to arouse students' interest in learning. So understanding students' schemata is necessary for locating the suitable content for the second class."Fig. 1"

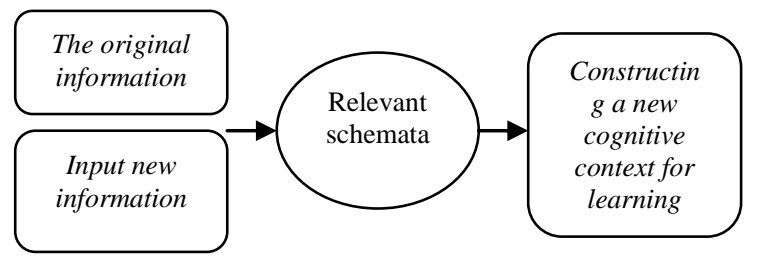

Fig. 1. Students' schemata.

\section{B. Analyzing the Students' Schemata and Constructing the Second Classroom of Listening, Speaking, Reading and Writing}

Teachers should make feasible plan to know students' English knowledge structure. In teaching, people also advocate individualized teaching. So for the teaching object, teachers should generally understand the basis of their advantages and disadvantages, in order to take the right teaching. Thus, at the beginning of the first term, students are required to take a large-scale competition in terms of listening speaking reading and writing skills thoroughly [5]. The exam can conduct a comprehensive test of their learning levels, and then teachers can analyze the language conditions of students, including the language strengths and weaknesses, and help students develop workable English learning programs which can provide constructive suggestions for the second classroom activities.

The teaching in the second English class can be divided into three levels of basic module, enhancing module, application module. In the basic module, teachers should lay emphasize on the solid English foundation for the students, including students' pronunciation, vocabulary, grammar and learning strategies. In this module, teachers should combine a lot of English textbooks, for example, to carry out the text reading, reciting, words competition, which helps to explore the best strategy for the students' learning. Then, after the constantly update and careful preparation of a whole semester, students should make great progress in the English foundation. Then, students are required to take an exam. If the vocabulary can reach to more than 2000, and the pronunciation can improved, they can enter into second module to enhance the learning level. In this module, teachers should lay emphasize on the students' communicative ability, the output. For example, watching movies in the classroom, actually, students are required to watch a movie again and again by imitating the protagonist's tone of voice, and then role-play, and finally giving the written summary or a open performance. After a period of preparation, students' vocabulary can reach to more than 3000, there is strong interest and confidence in English, so students can go to the application module in the second English class learning. In the third module, teachers should pay attention to not only the language output but also the application in practice. In the activity design, combining English activities with their major can activate the students' language schema and the students' professional schema as well. "Fig. 2"

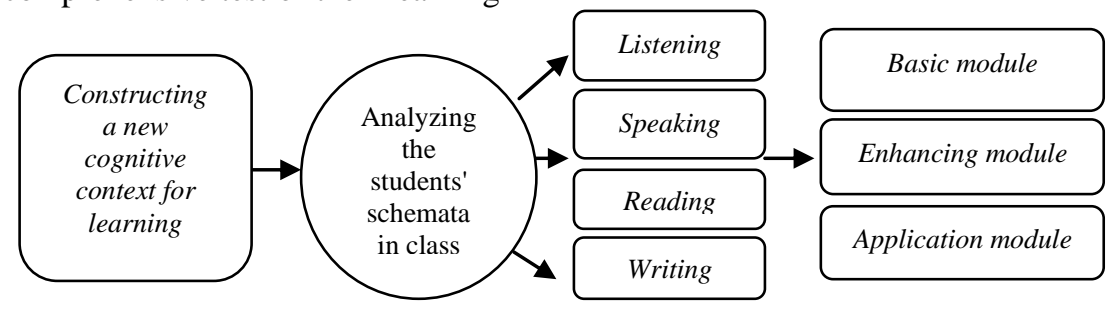

Fig. 2. Constructing a new cognitive context for learning.

\section{Constructing Stereoscopic Schema}

Language is the carrier of culture, and the cultural background of the English-speaking countries and China have a lot of difference, so teachers should pay more attention to the cultural background to broaden the students' knowledge, filling the deficiencies and the lack of knowledge of western culture [6]. According to schema theory, people can understand and connect the concept of input information and original information together. If students are lack of the background knowledge, they cannot construct effective cognitive schemata. Any language is cannot learned without culture. So teachers should construct language schema to help students not only to understand the pronunciation, intonation, spelling, but also the culture. Thus, the input schema is not simple equivalence of the speech sound, intonation and spelling, but the cultural schemata, three-dimensional schema construction of language and culture as a whole."Fig. 3" 


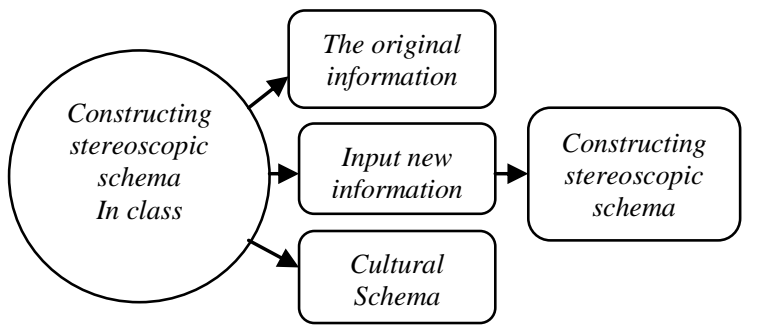

Fig. 3. Stereoscopic schema.

\section{THE FEASIBILITY AND SIGNIFICANCE OF DYNAMIC EVALUATION}

Dynamic teaching evaluation is the evaluation of students' learning process, which is based on the development of students' learning ability. In the course of the second English class, it is necessary and feasible to take a dynamic evaluation.

\section{A. The Dynamic Evaluation of the Second English Class Is Feasible}

Firstly, based on the dynamic evaluation of classroom activities, although compared with other courses, courses in English concepts and theories more and more abstract and difficult for students to understand, the discussion in the language classroom is still feasible. After entering the universities or colleges, students have a certain language ability, teaching and discussion in the language classroom will not only practice the ability to use the language, but also can deepen students' understanding of the language, cultural understanding. The arbitrariness of language symbols and rules about the relationship between the function of language, for example, the origin of language, language structure, language culture and the relationship between thinking and application which can be discussed in class in the forms of various class activities in the second English class. The dynamic evaluation can help students show interest, attitude, participation degree, judge the learning situation, encourage the English practice in their learning, as well as the continuous evaluation of their following learning development. This dynamic evaluation can provide feedback to the learning of students and teaching of teachers, so as to adjust section of teaching and learning.

Secondly, the dynamic evaluation of the second English class is feasible for the improvement of students' abilities in the English class. The various activities in the second English class is useful for the students learn English, not only listening and reading, but also speaking and writing, not only knowing the theory but also learning to solve practical problems and even cultivating students' thinking in the English logic way, which is necessary for students to practice their practical ability. It is not only necessary, but also feasible. Taking the activity of writing as an example, the teacher can let students learn about content to write. To cultivate students' ability of collecting data, sorting data. By recording the activities of students outside the classroom, learning results and interview and discussion, the process of learning can be observed, evaluated and monitored. Thus, the process of guidance and evaluation is a dynamic process.

\section{B. The Dynamic Evaluation Has Very Important Significance}

The dynamic evaluation of courses in the second English class is the evaluation of the learning processes not just the test and its result, especially the continuous evaluation of students' learning — not only evaluate the students' mastery of knowledge, but also evaluate in their daily learning process, such as achievements and feelings, the reflected attitude and learning strategies and other aspects of the situation [7]. Thus, the main elements of the evaluation are the students' learning interest and attitude, the sense of participation and involvement in the process of learning, intellectual development, comprehensive quality and the formation of values in the process of learning, which are important factors to learn the course, and these factors are can neither be measured in a quantitative way, nor be reflected in the summative assessment. In addition to non-intelligence factors, dynamic evaluation pays more attention to the students' training of innovation ability and practice ability, especially pay attention to the students' abilities on the application, analysis, synthesis, intelligence and high level of creative thinking. The emphasis on the evaluation of students' learning process causes students to attach importance to the daily performance of the learning process, the achievements and the emotion, reflecting the attitude of development strategies, which shows the concept of "student-centered" and "people oriented" idea[8]. Therefore, the dynamic evaluation can reflect the complex, invisible learning results and learning skills which the summative assessment can not; it can give full play of the students' learning enthusiasm, initiative and creativity, which conform to the goal of cultivating English speaker and user, conform to the development trend of modern language learning.

\section{The SPECIFIC PRACTICES OF DyNAMiC EVALUATION}

In order to achieve the goal of the second English class, people must take a variety of evaluation mechanism of integration and give full play to the wash back of the evaluation for the lifelong learning and lay the foundation for the English learners. In the traditional summative assessment, teachers should play a positive role of dynamic elements in the evaluation, and make the combination of the summative evaluation and dynamic evaluation, which can reflect the student's progress and experience in the process of learning in the dynamic learning process.

On the one hand, in the summative evaluation, teachers should first of all pay attention to the testing proportion of knowledge and ability, such as the subjective and objective questions in the test. At present, teachers should pay more attention to the ability in the exam not just the knowledge, for example, judge questions and analysis. In addition to let students make judgment on the item itself, if students combined with examples are analyzed, especially the analysis with examples of native language, which is a kind of ability assessment. Moreover, terminology, if students combined with examples to explain, especially with Chinese language examples to explain, the student will not be passively learned, they can learn it by heart. Secondly, the content of the examination should pay attention to be open, 
not only the content of the textbook but also the material around students' life, by examining the students' language competence instead of the students' memory. It is necessary to increase the number of problems of application and analysis, so that the proportion of the objective and subjective questions are more reasonable. The test questions application and analysis can promote students to start from the analysis of the language phenomenon and solve the problem as a learning goal, therefore, the combination of the language test and living language practice can promote more emphasis on capacity assessment, which is a kind of dynamic evaluation.

On the other hand, formative evaluation including selfevaluation, group evaluation and teacher evaluation. The self-evaluation, group evaluation has a great advantage in the second English class. The guidance of teachers should be discussed based on group activities in class discussion, so the focus of the evaluation should still be teachers. But the teachers' evaluation and summative evaluation in teacher evaluation is not the same. The teacher evaluation in the formative evaluation is based on the evaluation of the process, reflecting not only in the classroom discussion, classroom work and homework, but also in the subject research and writing papers, so it is a dynamic, continuous process. The emphasis should be placed on students of the phenomenon of language collection, analysis and use of language, which is also the student's ability on observation language, language analysis. In addition, formative evaluation can not be a single formality, so the evaluation should be fine, clear, and in the comprehensive consideration of students' statements, answering questions and writing on the subject, and the specific proportion should also be clearly defined; moreover, the evaluations of each part should have a certain objective basis, and the teacher must do the work with the concrete record, in order to ensure the rational and substantive points from the teachers. The teachers should make the records of students' ability on the analysis of the language phenomenon, focus on testing students' ability to combine the language practice and linguistic theory and the ability to apply linguistic theory to explain the phenomenon of language. The evaluation of teachers on the individual student is not only with other students by horizontal comparison; more important is to continue to examine the students' development process from the view of vertical way. From the perspective of development, taking the students' ability as the starting point and ultimate goal, the evaluation should be dynamic and developing.

\section{CONCLUSION}

The teaching evaluation in the second English classroom should adhere to the student-oriented, ability based rules, taking comprehensive examination, achieving the ideological nature, the unity of science and technology, playing the right guiding role of the examination. It is beneficial to cultivate and stimulate the students' interest in learning, and build the learning achievement and self-confidence, improve the comprehensive ability of using language. Therefore, it requires that the use of dynamic evaluation, the unite of formative assessment and summative assessment, can give full play to the advantages of both evaluation, and make reasonable evaluation of students, in order to achieve diversity, impartiality, comprehensive and comprehensive in teaching evaluation.

\section{REFERENCES}

[1] Luo Jing. Model of Class Stratification in College English Second Classroom Based on Classroom Teaching[J]. Journal of Harbin Vocational and Technical College, 2009 (06)

[2] Wang Jinqing. Schema Relevance Theory and English Writing in the Context of Cognitive Linguistics[J]. Journal of Hunan Medical University, 2009(3)

[3] Shi Rui. Preliminary Study of Textual Coherence From the Perspective of Cognitive Context[J]. Journal of Mudanjiang Normal College, 2009 (4)

[4] Tan Jinwen. Construction of Cognitive Context[J]. Journal of Fuyang Normal College, 2004 (02)

[5] Ur, P.A. Course in Language Teaching: Theory[M]. Practice and Cambridge: Cambridge University Press, 1996

[6] Liu Xubo. On Studey of Extra-curricular Teaching of Vocational College English From the Perseptive of Cognitive Context[J]. Nanchang Institution of Education, 2012(4)

[7] Yu Yunwei. Study on Dynamic Evaluation of English Linguistics[J]. The Foreign Language, 2010(1)

[8] Wu Xiulan. Summary of Formative Assessment in Foreign Language Teaching in Domestic Universities[J]. Foreign Language Circles, 2008(3) 